\title{
Implementing IVHM on Legacy Aircraft: Progress towards identifying an Optimal Combination of Technologies
}

\author{
Manuel Esperon-Miguez ${ }^{1}$, Ian K. Jennions ${ }^{2}$, Philip John ${ }^{3}$
}

\begin{abstract}
The aim of Integrated Vehicle Health Management (IVHM) is to improve the management of maintenance operations through the implementation of health monitoring tools on key components either by diagnosing deterioration or by estimating Remaining Useful Life (RUL) so as to effect timely, and cost effective, maintenance. Regarding the use of IVHM technology in legacy aircraft, one has to keep in mind that hardware modifications to improve the reliability of components is not normally considered a viable alternative to diagnostic and prognostic tools due to high certification costs. At the same time, the data and expertise gathered over years of operating the aircraft help to estimate much more accurately how different health monitoring tools could impact maintenance activities. Consequently, selecting the optimal combination of health monitoring tools for legacy aircraft is significantly easier than for a new design. While computer simulations of the maintenance process are essential to determine how different IVHM tools generate value for the stakeholders, it is not practicable to simulate all possible combinations in order to select which tools are to be installed. This paper describes a process to reduce their number of toolsets to be simulated starting with the identification of those components that present a higher potential to reduce maintenance costs and times in case their faults could be detected and/or predicted. This is followed by the definition of the minimum required accuracy of diagnostic and prognostic tools for each component. This enables designers to determine which tools -available or still being developed-can be implemented to achieve the expected improvement in maintenance operations. Different combinations of IVHM tools are then subjected to a preliminary risk and cost-benefit analysis. A significantly reduced number of combinations are then simulated to select the optimal blend of technologies.
\end{abstract}

\footnotetext{
${ }^{1}$ Manuel Esperon-Miguez ( $\left.\square\right)$

IVHM Centre, Cranfield University, Cranfield, MK43 0FQ, Bedfordshire, United Kingdom e-mail: m.esperonmiguez@cranfield.ac.uk

${ }^{2}$ IVHM Centre, Cranfield University, Cranfield, MK43 0FQ, Bedfordshire, United Kingdom

${ }^{3}$ School of Engineering, Cranfield University, Cranfield, MK43 0AL, United Kingdom
} 


\section{Introduction}

Integrated Vehicle Health Management (IVHM) aims to maximise the use of an asset and reduce its through life maintenance cost through the implementation of health monitoring tools that generate information regarding the condition of multiple components. This information is generated by either diagnostic or prognostic tools. Diagnostic tools reduce the time necessary to detect and isolate a fault, and can be used to avoid human error in the identification of faulty components. Prognostic tools estimate the Remaining Useful Life (RUL) of the component which, at least, helps to avoid a failure during a flight, allowing for the mission to be completed successfully and avoiding any secondary damage. If an accurate prognosis can be generated with enough time in advance (a.k.a. prognostic window or lead time), the replacement of the component can be scheduled at a time and location that minimises -or avoids- any disruption in the operation of the vehicle.

The implementation of IVHM technology has traditionally followed a reactive approach according to which a health monitoring tool is developed individually and, once its performance has been tested, it is put into service. There are two explanations for this approach: on one hand diagnostic and prognostic algorithms and the hardware necessary to implement them are normally developed independently by teams with expertise in the component/system being monitored; on the other hand, organizations lack a high level IVHM policy or program that requires a comprehensive analysis of the optimal combinations of tools to be developed and implemented. Consequently, aircraft end up with an eclectic set of IVHM tools that improve the maintainability of each part, but may have a negligible effect on the availability of the fleet.

However, it must be noted that the lack of a systems approach to IVHM implementation is not caused by lack of competence or vision. The use of several tools on a given aircraft results in interactions that must be carefully studied to ensure objectives are reached and their performance not undermined by overseeing critical interdependencies. From a maintenance perspective it is essential that the selection of components to be monitored takes into account their failure/replacement frequency, replacement time, delays and how IVHM can affect them. Given the complexity of maintenance operations this problem must be studied using computer-based simulations. From an implementation perspective, the interactions between tools can result in unforeseen problems with the hardware and/or the software. Thus, implementing and IVHM system that comprises diagnostic and prognostic tools that monitor several component becomes an engineering project that requires a significant investment and involves a great uncertainty.

Some methodologies to approach this problem do exist, but they normally focus on individual parts or a limited number of components or subsystems. It has been proposed to use Failure Modes, Effects and Criticality Analysis (FMECA) as the main basis for the design of full IVHM systems $[1 ; 3 ; 9]$. However, these methodologies, while applicable to a limited number of components, are not suita- 
ble for the analysis of a complete aircraft since it would be impractical to carry out a FMECA for each individual part, not to mention the analysis of all possible interactions between components and between their potential monitoring tools. As it is explained in the following sections, in the case of legacy aircraft, their unique combination of abundant historical maintenance data and constraints that rule out significant modifications of their systems, allow for a series of quantitative analyses leading to an optimal combination of diagnostic and prognostic tools.

Although IVHM can include the use of tools to improve the management of logistics, maintenance and operations, this paper discusses a methodology to select the optimal combination of diagnostic and prognostic tools by performing different quantitative analyses before defining the final set of tools based on the results obtained from a computer-based simulation of maintenance activities. Consequently, in this text the use of the terms "health monitoring tools" or simply "tools" makes reference to diagnostic or prognostic tools.

\section{IVHM and legacy systems}

Retrofitting IVHM into legacy platforms presents a very specific set of challenges that must be acknowledged from the beginning. While some of these issues affect all kinds of aircraft, they are more acute for aircraft that have been operated for years but are no longer being manufactured. A short list and discussion are presented bellow to show the breadth and depth of these issues.

Technical constraints: Geometric and weight constraints can result in the need to make changes to the structure or other components to accommodate new sensors, wires, electronics, etc. However, the cost of certifying the new tools and any changes required can exceed that of the design, manufacturing and installation of the necessary hardware. The need to ground the aircraft to install and test any new IVHM tool can disrupt normal operations and result in a loss of revenue, making these modifications even more difficult to justify. Software faces similar challenges given the critical role it plays nowadays both on-board and off-board [4]. The cost of certifying major hardware modifications and the uncertainty of potential benefits undermine the implementation of IVHM technology on legacy aircraft [2]. Consequently, for health monitoring tools to be implemented they must require very small or no modifications of existing systems.

Role of organizations: The implementation of IVHM has a significant impact on each stakeholder's organization and vice versa. Aircraft will have to remain grounded for a significant amount of time resulting in significant disruptions in normal operations [4]. Moreover, in order to maximise the benefit of this technology maintenance practices have to change to be able to act based on the information provided by the new health monitoring system. Cultural barriers such as lack of understanding of the real benefits of IVHM and insufficient management support can jeopardize its development and put in service [7] [11]. 
Regulations and standards: Maintenance organizations normally have some Condition-Based Maintenance (CBM) policies already in place. Depending on the aircraft, the organization it belongs to and its area of operation some of these procedures can be regulated and made compulsory. As a consequence, a prognostic tool that monitors a component for which CBM is compulsory is not likely to be justifiable from an economic stand point since the investment will not be translated into a significant saving [5]. Therefore, special attention must be paid to maintenance regulations and standards.

Historical Maintenance Data: What sets legacy aircraft apart is the amount of information regarding the reliability of their components, operational environment, maintenance processes and failure modes. While new aircraft rely on estimates based on their design characteristics or a few tests, legacy aircraft present much more comprehensive datasets with information gathered in real operational conditions. As a result, not only is there more information available, but also it is much more accurate.

Although there is a lot of information recorded in maintenance and mission $\operatorname{logs}$ it can be difficult to transform it into useful data for the development of an IVHM system. Analysing records kept in handwritten documents or early databases can become an arduous task. Nevertheless, this still represents a significant advantage over new aircraft and, as will be discussed in the following sections, proves crucial in the selection of the optimal combination of health monitoring tools to be retrofitted on a given aircraft.

\subsection{Identifying the role of stakeholders}

Given the complexity of the aviation industry nowadays, the role of different stakeholders must be identified from a very early stage. Whereas in the past the owner, operator and maintainer of a fleet were the same entity, outsourcing and leasing have generated all sort of different sources of revenue, but also makes it difficult to pinpoint who should pay for the development of IVHM technology. Furthermore, health monitoring technology can underpin the transformation of manufacturers to service providers, meaning any CBA for IVHM must take into account the effect it can have on current and future contracts as well as the company's mid and long-term strategy [8]. Wheeler et al. [13] identified the goals for different stakeholders according to their responsibilities: logistics, mission operation, maintenance and fleet management. These goals are then divided into those which can be achieved using diagnostic tools and those which need the use of prognostic tools. 


\subsection{Framing the problem}

The fact that major modifications of a legacy aircraft's systems are too expensive represents an advantage compared to new aircraft for which this is a viable option. For legacy aircraft, the business case for an IVHM system to monitor a certain group of components is very easy to justify when faced with the option of modifying such components to improve their reliability and maintainability to a level that results in the same improvement in cost and availability. Consequently, these limitations can be seen as the constraints for a mathematical problem in which major changes in an aircraft's systems are no longer an option. As a result, it can be assumed that the performance of the aircraft is not going to be affected, nor will its interdependencies between systems.

Computer simulation of aircraft maintenance systems can be used to study how health monitoring technology affect maintenance activities and, consequently, maintenance cost and availability at aircraft and fleet level. Unlike aircraft that are being designed or have only been operated for a short period, legacy aircraft can rely on historical maintenance data to provide all the information necessary for the development of these models.

In summary, the use of historical maintenance data in combinations with the constraints just mentioned helps to formulate accurate CBAs for IVHM systems for legacy aircraft.

\section{Quantifying the benefits of IVHM}

IVHM affects both maintenance costs and times. Consequently, the availability of an aircraft -and the squadron and fleet to which it belongs- will depend on the tools that form such an IVHM system. Not only does health monitoring reduce the time necessary to replace a component by performing faster diagnoses or avoiding secondary failures, but it can also affect the timing of, and location for, maintenance actions. Taking into account that several tasks are performed simultaneously during both scheduled and unscheduled maintenance stops, it is not possible to calculate analytically the duration of each stop. Furthermore, delays play a major role in maintenance and can be due to different logistic, administrative or technical causes. The fact that maintainers organise maintenance tasks depending on operational demands and the minimum equipment lists for future missions only increases the complexity of the problem. It is only through the use of computerbased simulations of maintenance activities that these complexities can be captured and the effect of IVHM technology estimated quantitatively.

The development and validation of these models requires significant amounts of data. To ensure the benefits of implementing IVHM are estimated correctly these datasets must include, not only the average of variables such as Mean Time 
Between Failures (MTBF) or Mean Time To Replace (MTTR), but also their variances.

Evidently, the model must take into account the effect any potential diagnostic or prognostic tool can have on maintenance costs and times as well as availability. In order to do so it is essential to acknowledge that health monitoring tools are not $100 \%$ accurate. Diagnostic tools can produce false positives (a.k.a. false alarms) by indicating a healthy component has failed, or false negatives if a faulty component is not detected. Similarly, prognostic tools estimate the RUL of a component at certain point in time and its replacement is scheduled according to that estimate, but if the estimation is too optimistic it might have to be replaced at a less convenient time and location or even fail during a flight. Being able to simulate the performance of health monitoring tools is essential to compare tools with lower cost and performance with more reliable and expensive ones.

\subsection{Reducing the number of runs}

Ideally, once the maintenance model has been developed and validated, different combinations of diagnostic and prognostic tools can be tested on it. However, while the computer model is the only way to carry out a solid CBA, it is not practical - or even possible - to simulate the effect of all potential combinations. Taking into account that aircraft comprise thousands of components, a comprehensive analysis of all options should consider, at least, a few dozen components to be monitored, even if the final number of tools to be implemented may be lower. For example, if 10 tools are to be chosen out of 50 possible options, this represents more than 10 billion possible combinations. Even taking into account incompatibilities between tools due to conflicts caused by their hardware or software, it is unlikely that the total number of toolsets is reduced significantly enough so all combinations can be studied and compared thoroughly.

Consequently, there is a need for a methodology to reduce the number of combinations of diagnostic and prognostic tools whose impact on maintenance cost and availability is to be studied using a computer simulation of aircraft maintenance activities. Such methodology must be based on a set of quantitative analyses to avoid any bias. Several combinations must be generated with this methodology to allow for sanity checks and to compare how they affect other factors apart from cost and availability. This methodology has been developed taking into account the constraints imposed on legacy aircraft, the availability and accuracy of historical maintenance data and the information that can be gathered at the conceptual design stage on the characteristics and performance of health monitoring tools. The main steps, which will be discussed in detail in the following sections, are: 
1. Identify components more likely to have their maintenance time and cost reduced if monitored.

2. Select a preliminary list of health monitoring tools capable of detecting or predicting the failure of the components previously selected.

3. Identify incompatible combinations of tools due to software or hardware conflicts.

4. Preselect toolsets according to their expected Return On Investment (ROI) and financial risk.

\subsection{Identifying critical components}

The first step to reduce the number of simulations necessary for a comprehensive comparison of all the alternatives for an IVHM system involves identifying which components should be monitored. At this phase the number of components preselected is larger than the number of parts that will finally be monitored to allow for modifications in later stages. The objective is to identify which components are more likely to reduce maintenance time and cost if they are monitored by a diagnostic or a prognostic tool.

It is easy to evaluate what is the cost of replacing each component per flying hour as well as its corrective or preventive maintenance time per flying hour. Diagnostic tools essentially reduce the time dedicate to fault identification and isolation which will only affect labour costs. A prognostic tool affect the probability of a component having to be replaced at different locations (affecting logistic delays and shipping costs) and whether it will be an unscheduled task or part of a scheduled maintenance stop (with different costs and delays).

A method proposed in the past consists of analysing the possible outcomes of failure using Event Tree Analysis (ETA) [6] (Figure 1). Using the probability of a certain component failing as starting point, the tree forks based on the outcome of using a certain type of IVHM tool. A long-term prognostic tool can provide a RUL with a prognostic window long enough to schedule the replacement of the part so it will not affect the inherent availability of the aircraft. However, the estimated RUL can be incorrect. In that case there is the possibility to use a short-term prognostic algorithm and replace the part during an unscheduled stop, avoiding a possible mission loss or even secondary damages. Nevertheless, this recalculated RUL can also be overly optimistic, meaning the failure will take place and will need to be detected and isolated. If a diagnostic tool is fitted this can be performed automatically, but there is always the possibility of a false negative resulting in a longer time to diagnose the fault. The tree also includes the possibility of a healthy component being flagged as faulty by a diagnostic tool.

The order in which these tools appear in the tree does not reflect how health monitoring algorithms operate, it simply indicates in which order they will define 
the final outcome. Additionally, the fact that three kinds of tools are included in the tree does not imply that each component counts with all of them.

One of the advantages of this setup is that it accounts for the fact that some components can utilise some diagnostic capability in the form of Built-In Test Equipment (BITE) or be replaced according a preventive maintenance scheme, which, for the purpose of the ETA, has the same effect as a prognostic tool. Since it is possible to upgrade a health monitoring tool, there is no reason to exclude them from this analysis.

\begin{tabular}{|c|c|c|c|c|c|}
\hline \multirow[b]{5}{*}{$P_{F}$} & \multicolumn{3}{|c|}{ Detectability with IVHM } & \multirow{2}{*}{ Cost } & \multirow{2}{*}{ Time } \\
\hline & $\begin{array}{l}\text { Long Term } \\
\text { Prognosis }\end{array}$ & $\begin{array}{c}\text { Short Term } \\
\text { Prognosis }\end{array}$ & Diagnosis & & \\
\hline & $1-\mathrm{P}_{\mathrm{LP}}$ & & & \multirow{2}{*}{$\mathrm{C}_{\mathrm{LP}}$} & \multirow{2}{*}{$t_{L P}$} \\
\hline & \multirow{2}{*}{ SUCCESS } & \multirow[b]{2}{*}{$1-\mathrm{P}_{\mathrm{SP}}$} & & & \\
\hline & & & & \multirow{2}{*}{$\mathrm{C}_{\mathrm{SP}}$} & \multirow{2}{*}{$t_{S P}$} \\
\hline & $\mathrm{P}_{\mathrm{LP}}$ & SUCCESS & \multirow[b]{2}{*}{$1-\mathrm{P}_{\mathrm{FN}}$} & & \\
\hline & \multirow[t]{5}{*}{ FAILURE } & $\mathrm{P}_{\mathrm{SP}}$ & & $C_{D}$ & $t_{D}$ \\
\hline & & FAILURE & $\mathrm{P}_{\mathrm{FN}}$ & $C$ & \\
\hline & & & FAILURE & $C_{F N}$ & $t_{\mathrm{FN}}$ \\
\hline \multirow{4}{*}{$1-\mathrm{P}_{\mathrm{F}}$} & & & 1-P $\mathrm{P}_{\mathrm{FA}}$ & \multirow{2}{*}{0} & \multirow{2}{*}{0} \\
\hline & & & SUCCESS & & \\
\hline & & & $\mathrm{P}_{\mathrm{FA}}$ & \multirow[t]{2}{*}{$\mathrm{C}_{\mathrm{FA}}$} & \multirow[t]{2}{*}{$\mathrm{t}_{\mathrm{FA}}$} \\
\hline & & & FAILURE & & \\
\hline
\end{tabular}

Figure 1 ETA for the use of health monitoring tools on a single component.

Analytical equations for the maintenance time, $T$, and costs, $C$, incurred per flying hour for each component are easy obtained based on this ETA [6]. Since they are polynomial expressions the derivatives can also be calculated analytically quite easily.

$C=P_{F}\left(\left(1-P_{L P}\right) C_{L P}+P_{L P}\left(\left(1-P_{S P}\right) C_{S P}+P_{S P}\left(\left(1-P_{F N}\right) C_{D}+P_{F N} C_{F N}\right)\right)\right)+\left(1-P_{F}\right) P_{F A} C_{F A}{ }^{(1)}$

$T=P_{F}\left(\left(1-P_{L P}\right) t_{L P}+P_{L P}\left(\left(1-P_{S P}\right) t_{S P}+P_{S P}\left(\left(1-P_{F N}\right) t_{D}+P_{F N} t_{F N}\right)\right)\right)+\left(1-P_{F}\right) P_{F A} t_{F A}$

where the performance of long and short term prognostic tools is defined by $P_{L P}$ and $P_{S P}$, respectively; and the probability of false alarms and false negatives by $P_{F A}$ and $P_{F N}$ respectively. If a long term prognostic tool works correctly the cost and time of replacing the components are $C_{L P}$ and $t_{L P}$ respectively, and $C_{S P}$ and $t_{S P}$ in case a short term prognostic tool is used. If the fault is detected by a diagnostic tool the cost and downtime will be $C_{D}$ and $t_{D}$. For false alarms costs and downtimes are denoted by $C_{F A}$ and $t_{F A}$, and by $C_{F N}$ and $t_{F N}$ for false negatives.

This ranking takes into account the maintenance time spent on individual components. As it has been discussed previously, there is not a direct correlation be- 
tween the reduction of maintenance time of certain individual parts and the availability of the aircraft. However, components with longer maintenance times and higher sensitivities to the use of IVHM are more likely to have an important role in the improvement of the availability of the fleet. Once the components have been ranked the computer model can be used to verify which of those at the top of the list are responsible for most of the unscheduled maintenance stops and delays.

Identifying which components are the best candidates to be monitored by diagnostic and prognostic tools is useful, but it is not the kind of information that can be used to run computer simulations. The model uses the performance of health monitoring tools, meaning tools capable of assessing the condition of these top components have to found, as explained in the following section.

\subsection{Performance requirements for a preliminary selection of health monitoring tools}

Once key components have been identified it is necessary to find which tools can be used to monitor them. Original Equipment Manufacturers (OEMs), companies specialised in health monitoring technology and universities can be contacted to determine which tools are available or can be developed.

Even at such an early stage in the design of an IVHM system it is necessary to define basic technical and economic requirements to be able to compare different toolsets. Once again, the computer model is essential to define the minimum expected reductions in maintenance times and costs for each component to achieve the desired availability and total maintenance cost.

As shown in Eq. 1 and 2, it is possible to define the maintenance cost, and time of a component as a function of the performance of different health monitoring tools. If cost and time become a design requirement ( $C^{*}$ and $T^{*}$ respectively) these equations can be used to define the required performance for a diagnostic (Eq. 3 to 6) or prognostic tools (Eq. 7 to 9).

The progress in health monitoring technology has not been homogeneous for all kind of systems and it is possible that for certain components diagnostic or prognostic tools that satisfy the performance requirements are not available yet. The possibility of developing a new tool, or improving on an existing one, should be studied at this stage. Conversely, it is also possible that for other components several candidates can be identified. Rather than select a single tool for each component by a process of elimination, all possible options should be considered. The following sections illustrates how the interactions between tools can be studied to identify which components should finally be monitored and by which tool. 


$$
\begin{aligned}
& \begin{array}{c}
\text { Diagnostic } \\
\text { Tools }
\end{array}\left[\begin{array}{c}
P_{F A} \geq 0 ; P_{F N} \geq 0 \\
P_{F A} \leq \frac{C^{*}-P_{F}\left(\left(1-P_{F N}\right) C_{D}+P_{F N} C_{F N}\right)}{\left(1-P_{F}\right) C_{F A}} \\
P_{F A} \leq \frac{T^{*}-P_{F}\left(\left(1-P_{F N}\right) t_{D}+P_{F N} t_{F N}\right)}{\left(1-P_{F}\right) t_{F A}}
\end{array}\right. \\
& P_{L P} \geq 0 \\
& P_{L P} \leq \frac{\frac{C^{*}-\left(1-P_{F}\right) P_{F A} C_{F A}}{P_{F}}-C_{L P}}{\left(1-P_{F N}\right) C_{D}+P_{F N} C_{F N}-C_{L P}} \\
& P_{L P} \leq \frac{\frac{T^{*}-\left(1-P_{F}\right) P_{F A} t_{F A}}{P_{F}}-t_{L P}}{\left(1-P_{F N}\right) t_{D}+P_{F N} t_{F N}-t_{L P}}
\end{aligned}
$$

\subsection{Uncertainties and their effect on CBAs for IVHM}

Most parameters in maintenance activities are normally random variables due to the fact that even repetitive tasks seldom take the same amount of time or require the same amount of attention and resources. It is possible to work with average values for some basic analyses, but if the objective is to ensure availability stays above a certain value and maintenance costs do not exceed a given limit working with average values results in a 50\% chance of failing to reach the objectives.

The sources of uncertainty can be divided into two main categories. Epistemic, or systemic, uncertainties are caused by inaccuracies in the measurement, recording or modelling of a given parameter. These are the kind of uncertainties which affect the accuracy of maintenance records. To begin with, recorded times are never perfectly accurate but rounded to the nearest multiple of five, ten or fifteen minutes. Additionally, while the total maintenance time spent on each component is often recorded, this is not always the case for the different steps involved (e.g.: preparation, diagnosis, check-out, etc.) or the delays. Even in those few cases when records include this information values are most likely approximations written down after the work has been completed.

The second group comprises the sources of aleatoric, or statistical, uncertainties which are those caused by the random variation of parameters over time. Recurring costs, time spent on different activities, delays and the performance of health monitoring tools are the most prominent. While the amount a supplier charges for a part can be fairly constant (this does not apply to expensive components with 
low failure rates and low stock), shipping and storage costs can vary considerably. The same can be said about the time dedicated to maintenance tasks, whose variability is related to the complexity of the task.

The uncertainty of the performance of IVHM tools has been well documented. Lopez \& Sarigul-Klijn [10], showed how the reliability of an IVHM tool varies depending on the characteristics of the fault, which are different on every occasion, and this translates into uncertainty about its performance. Furthermore, Saxena et al. [12] also analysed how the accuracy of prognostic algorithms evolves with time, with the RUL becoming more accurate as the component approaches its point of failure.

As a result, engineers who define the performance requirements not only must acknowledge that expected maintenance costs and times follow probability distributions but also take into account that the variance of the performance of each tool must be below a certain threshold. This threshold can be defined using Eq. 1 and 2 as a basis to determine the variances of performance parameters:

$$
\begin{gathered}
\operatorname{Var}(C)=\operatorname{Var}\left(P_{F N} P_{F}\left(C_{F N}-C_{D}\right)\right)+\operatorname{Var}\left(P_{F} C_{D}\right)+\operatorname{Var}\left(P_{F A}\left(1-P_{F}\right) C_{F A}\right) \\
\operatorname{Var}(T)=\operatorname{Var}\left(P_{F N} P_{F}\left(t_{F N}-t_{D}\right)\right)+\operatorname{Var}\left(P_{F} t_{D}\right)+\operatorname{Var}\left(P_{F A}\left(1-P_{F}\right) t_{F A}\right)
\end{gathered}
$$

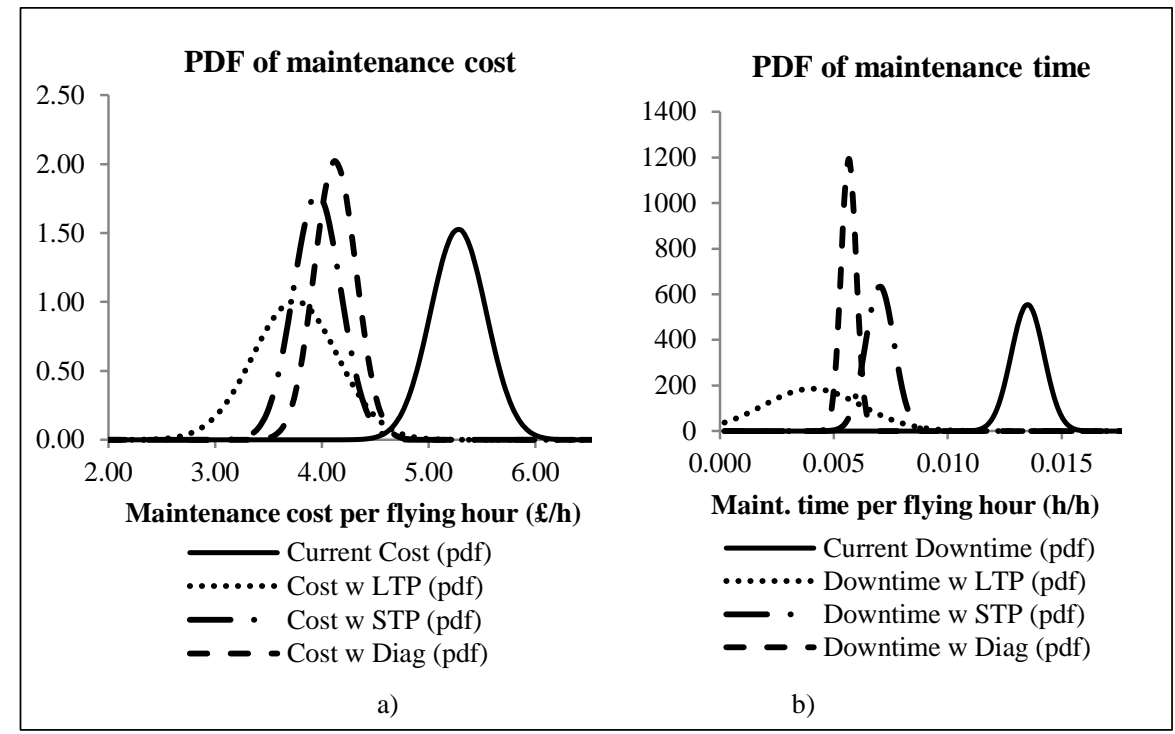

Figure 2 Examples of the effect of IVHM tools on the PDF of maintenance a) cost and b) time of a component.

Figure 2 shows the effect diagnostic tools, short term prognostic tools and longterm prognostic tools can have in the probability distributions of the maintenance cost and time per flying hour of a component. 
It would seem as if these uncertainties add complexity to our problem increasing the difficulty of finding an optimal combination of diagnostic and prognostic tools. However, as explained in the following section, these uncertainties can be used to carry out a risk analysis of the different sets of tools and to reduce the number of combinations that should finally be studied using computer simulation.

\subsection{Balancing ROI and risk}

Comparing toolsets must take into account the possibility of sharing resources between tools in their design, testing, manufacturing, implementation and operation. In other words, tools can share -among others- sensors, memory, flight test expenses, recurring costs, etc. This translates to a reduction in the investment necessary to put a certain group of tools in service. Consequently, the ROI of each toolset is not the weighted average of the ROIs of those tools it comprises, but the ratio between the sum of their expected profits and the total cost of developing, implementing and operating the complete IVHM system. This profit is essentially based on the costs avoided thanks to the use of a certain health monitoring tool, but other benefits can be included. In mathematical terms, for a toolset with $n$ tools in which the project budget for each tool has been divided into $\mathrm{m}$ phases or parts this can expressed as:

$$
R O I=\frac{\sum_{i=1}^{n} P_{i}}{\sum_{i=1}^{n} C_{i}}=\frac{\sum_{i=1}^{n} P_{i}}{\sum_{i=1}^{n} \sum_{j}^{m} c_{i j} / \alpha_{i j}}
$$

where $P_{i}$ is the expected profit from tool $i$; $C_{i}$ the total cost of tool $i$; $c_{i j}$ the cost of tool $i$ for part $j$ of its budget; and $\alpha_{i j}$ the number of tools with which $c_{i j}$ is shared.

However, sharing resources means that a deviation in their cost can effectively raise the cost of several tools. For example, if algorithms are processed in a centralised unit whose costs exceeds the original budget this will also impact the cost of each individual health monitoring tool. A federated IVHM system with algorithms run in individual processing units may be more expensive, but its total cost is less vulnerable to this kind of problems.

Comparing toolsets becomes even more complicated when options include tools that are under development and not fully proven. Mature diagnostic and prognostic tools are less likely to present problems and have significant cost variations, but their performance can be lower than tools that are still being developed and employ the latest technology. The cost of the latter, however, is more likely to deviate from the budget.

This resembles a classic financial investment problem in which investors must select the optimal combination of assets to maximise the return of their portfolio while keeping risk within reasonable limits. As in the problem described in this article, financial assets have some degree of correlation and this must be carefully studied to avoid situations in which an investor can be severely affected by fluctu- 
ations in the market (e.g.: stock prices of logistic companies are affected by the fluctuation of oil prices in commodity markets, gold prices and the U\$D are normally inversely correlated, etc.).

There are all sorts of financial analysis tools that can be applied to solve this problem, but there is an important part of this financial analysis tools ignore: the variation of the ROI of each health monitoring tool depending on how it is combined with others. This is due to the fact that the return on a financial product is not affected by how much one invests in other assets.

Figure 3.a shows the result of using a tool known as the efficient portfolio frontier to analyse combinations of IVHM tools. As toolsets include larger numbers of diagnostic and prognostic tools the risk decreases because deviations in the cost of individual tools have a smaller impact on the total investment. However, the ROI tends to the average ROI of all possible options because the savings are not taken into account. Figure 3.b shows how the ROI can increase significantly if IVHM tools are combined appropriately.

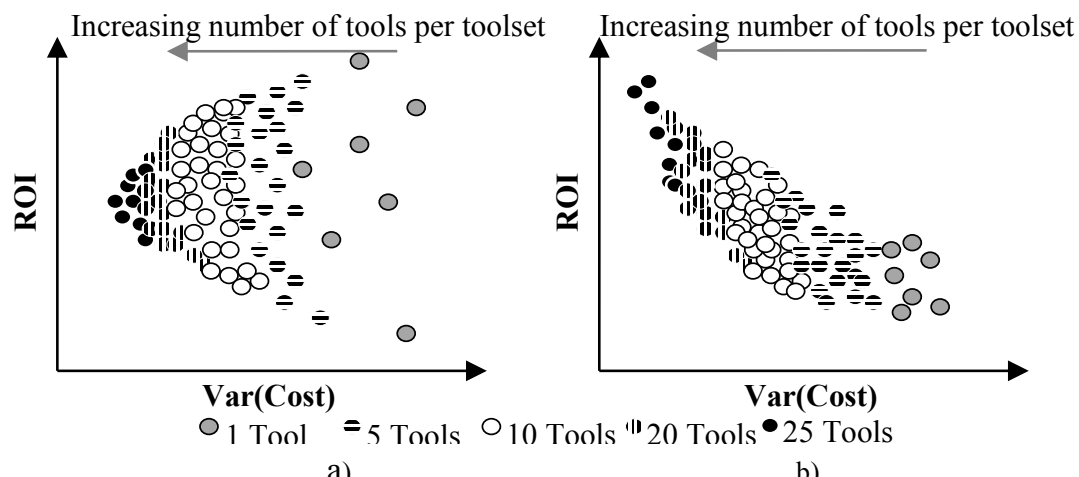

Figure 3 ROI vs. variance of cost for IVHM toolsets using financial analysis (a) and including shared costs (b)

Those toolsets that present a higher ROI and a lower variance of costs can be tested on the computer simulation. This will determine which combination of tools should be retrofitted on the aircraft and provide a much more accurate estimation of the final outcome.

\section{Conclusions}

The methodology presented in this paper illustrates how it is possible to carry out exhaustive quantitative analyses of the effect of retrofitting different IVHM toolsets on legacy aircraft without being overwhelmed by the number of options to compare. While computer simulations are essential to ensure CBAs for IVHM are 
accurate, they cannot be the only tool available to define the optimal combination of health monitoring tools.

Uncertainty plays a major role in the analysis and comparison of different toolsets. Design teams must be aware of the main sources or uncertainty and to what degree it affects the information generated at each stage of the process. Second order uncertainties or "uncertainty of uncertainties" is a major area of research IVHM developers cannot ignore. The trustworthiness of any CBA is directly affected by the variance of the variables it uses and to be able to define them a deep knowledge of aircraft design, maintenance and operations is required.

While financial analysis tools can be used to determine how risk changes depending on how diagnostic and prognostic tools are combined, they must be modified to take into account the effect potential savings have on the resulting ROI.

\section{Acknowledgements}

This work has been supported by the IVHM Centre at Cranfield University. The authors would like to thank the partners of the IVHM Centre for their support in this project.

\section{References}

1. Ashby, M. J. and Byer, R. J. (2002), "An approach for conducting a cost benefit analysis of aircraft engine prognostics and health management functions", Aerospace Conference Proceedings, 2002. IEEE, Vol. 6, pp. 6-2847.

2. Azzam, H., Beaven, F., Gill, L. and Wallace, M. (2004), "A route for qualifying/certifying an affordable structural prognostic health management (SPHM) system", Aerospace Conference, 2004. Proceedings. 2004 IEEE, Vol. 6, pp. 3791.

3. Banks, J., Reichard, K., Crow, E. and Nickell, K. (2009), "How engineers can conduct costbenefit analysis for PHM systems", Aerospace and Electronic Systems Magazine, IEEE, vol. 24, no. 3, pp. 22-30.

4. Dunsdon, J. and Harrington, M. (2008), "The Application of Open System Architecture for Condition Based Maintenance to Complete IVHM", Aerospace Conference, 2008 IEEE, pp. 1 .

5. Esperon-Miguez, M., John, P. and Jennions, I. K. (2012), "The effect of current military maintenance practices and regulations on the implementation of Integrated Vehicle Health Management technology", A-MEST' 12, Seville, .

6. Esperon-Miguez, M., John, P. and Jennions, I. K. (2012), "Uncertainty of performance metrics for IVHM tools according to business targets", First European Conference of the Prognostics and Health Management Society 2012, Vol. 11, Dresden, pp. 11.

7. Grubic, T., Redding, L., Baines, T. and Julien, D. (2011), "The adoption and use of diagnostic and prognostic technology within UK-based manufacturers", Proceedings of the Institution of Mechanical Engineers, Part B: Journal of Engineering Manufacture, vol. 225, no. 8, pp. 1457-1470. 
8. Hess, A., Calvello, G., Frith, P., Engel, S. J. and Hoitsma, D. (2006), "Challenges, Issues, and Lessons Learned Chasing the "Big P": Real Predictive Prognostics Part 2", Aerospace Conference, 2006 IEEE, pp. 1.

9. Kacprzynski, G. J., Roemer, M. J. and Hess, A. J. (2002), "Health management system design: Development, simulation and cost/benefit optimization", Aerospace Conference Proceedings, 2002. IEEE, Vol. 6, pp. 6-3065.

10. Lopez, I. and Sarigul-Klijn, N. (2010), "A review of uncertainty in flight vehicle structural damage monitoring, diagnosis and control: Challenges and opportunities", Progress in Aerospace Sciences, vol. 46, no. 7, pp. 247-273

11. MacConnell, J. H. (2007), "ISHM \& Design: A review of the benefits of the ideal ISHM system", Aerospace Conference, 2007 IEEE, pp. 1.

12. Saxena, A., Celaya, J., Saha, B., Saha, S. and Goebel, K. (2010), "Metrics for Offline Evaluation of Prognostic Performance", International Journal of Prognostics and Health Management.

13. Wheeler, K., Kurtoglu, T. and Poll, S. (2009), "A Survey of Health Management User Objectives Related to Diagnostic and Prognostic Metrics". 
2013-10-30

Implementing IVHM on legacy aircraft: progress towards identifying an optimal combination of technologies

\section{Esperon Miguez, Manuel}

Springer

Manuel Esperon-Miguez, lan K. Jennions and Philip John. Implementing IVHM on legacy aircraft: progress towards identifying an optimal combination of technologies. Proceedings of the 8th World Congress on Engineering Asset Management (WCEAM 2013), 30 October - 1 November 2013, Hong Kong.

https://dspace.lib.cranfield.ac.uk/handle/1826/11577

Downloaded from Cranfield Library Services E-Repository 